Relations industrielles

Industrial Relations

\title{
Le Congrès canadien du travail
}

\section{Eugène Forsey}

Volume 4, numéro 1, septembre 1948

URI : https://id.erudit.org/iderudit/1023424ar

DOI : https://doi.org/10.7202/1023424ar

Aller au sommaire du numéro

\section{Éditeur(s)}

Département des relations industrielles de l’Université Laval

\section{ISSN}

0034-379X (imprimé)

1703-8138 (numérique)

Découvrir la revue

Citer cet article

Forsey, E. (1948). Le Congrès canadien du travail. Relations industrielles /

Industrial Relations, 4(1), 5-10. https://doi.org/10.7202/1023424ar

Tous droits réservés @ Département des relations industrielles de l’Université Laval, 1948
Ce document est protégé par la loi sur le droit d'auteur. L'utilisation des services d'Érudit (y compris la reproduction) est assujettie à sa politique d'utilisation que vous pouvez consulter en ligne.

https://apropos.erudit.org/fr/usagers/politique-dutilisation/ 
riodiques des partis politiques des temps modernes.

Etre constamment libre de toute attache politique est donc le parti le plus sage à prendre, pour faire avec autorité et influence les représentations nécessaires en faveur de la classe ouvrière et ainsi faire librement - ce qui est admis - de l'action politique non-partisane. C'est l'attitude de la C.T.C.C.

Elle entend demeurer aussi une force nationale. Elle croit que le syndicalisme canadien doit être cent pour cent autonome et régler ses propres affaires. Elle réprouve comme subversif aux intérêts nationaux bien compris toute subordination syndicale directe ou indirecte au syndicalisme américain.

Elle croit enfin que le syndicalisme se doit d'être intégralement national dans son origine, son inspiration et son orientation, afin d'adapter son action politique, économique et sociale suivant le meilleur intérêt de l'économie nationale, et ce, pour le bien général.

La C.T.C.C. s'est acquise une réputation d'intégrité. Sa voix est écoutée avec un intérêt croissant par les pouvoirs publics. Sa contribution à la restauration sociale chrétienne du pays est aux premiers rangs des avant-postes.

\title{
LE CONGRĖS CANADIEN DU TRAVAIL
}

\author{
Eugene Forsey
}

Le Congrès canadien du travail est le plus jeune et le moins considérable - pas tellement! des deux principaux organismes ouvriers au Canada. Le Congrès des métiers et du travail, dont la fondation remonte à 1873, comptait en 1946 quelque 356,000 membres. Le Congrès canadien du travail, lui, n'a pris corps qu'en 1940, et pourtant, dès 1946, il pouvait se réclamer d'environ 315,000 adhérents.

Les deux Congrès diffèrent donc et par l'âge et par les proportions. Mais à cela ne se limitent point les différences!

Et d'abord, soulignons les deux principes bien différents qui servent de base à leur organisation. Le Congrès des métiers et du travail groupe sous son égide quelques unions industrielle, v.g. l'International Ladies Garment Workers' Union et la Canadian Seamen's Union. Mais il rassemble la plupart de ses membres sous l'étiquette des unions professionnelles. Les plombiers appartiennent à des syndicats de plombiers, les charpentiers, les électriciens sont organisés comme tels, quelle que soit l'industrie où ils exercent leur métier. Le Congrès canadien du travail, au contraire, est constitué entièrement d'unions indus-

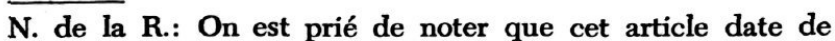
quelques mois, et ne tient pas compte des événements survenus depuis. trielles. Les travailleurs qui exercent leur activité dans l'industrie de l'acier sont organisés en tant qu'ouvriers de l'acier. Même chose pour les employés des établissements de salaison, les mineurs, etc. On ne tient pas compte de la tâche particulière qu'ils accomplissent.

Cette différence est extrêmement importante. C'est l'une des principales raisons pour lesquelles le C.C.T. est passé de 70,000 à 315,000 membres en six ans, alors que le C.M.T., durant la même période, n'a connu qu'une augmentation de 200,000 à 356,000 affiliés. L'organisation des ouvriers selon la profession ne convient plus à l'industrie moderne, où tout tend vers la production massive et où de puissantes corporations ont à faire face à des groupements nombreux d'ouvriers semi-qualifiés ou non qualifiés. La plupart de ces ouvriers n'ont pas de métier. Alors, tenter de les organiser sur une base professionnelle, c'est tout simplement vouloir opposer au puissant employeur un éparpillement de syndicats faibles et peu considérables, ou même rien du tout. Jusqu'à douze ans passés, les industries de production massive au Canada étaient pour ainsi dire complètement organisées. Le Rapport sur l'organisation ouvrière, présenté en 1935 par le ministère canadien du travail, n'inclut même pas les ouvriers de l'automobile, du fer, de l'acier et de l'étain, prédécesseurs des ouvriers unis de l'acier ou du bois. 
Jusqu'à l'année 1940, les syndicats des travailleurs des mines, des moulins et des fonderies ne comptaient que 176 membres, ceux des maisons de salaison 221, ceux du caoutchouc, 610 , et enfin, les électriciens, radio-techniciens et machinistes, 637. En 1946, les ouvriers de l'automobile formaient le plus puissant syndicat au Canada, avec 50,000 membres; les ouvriers de l'acier avaient un syndicat de 35,000 membres; le syndicat du bois groupait 27,000 membres, celui de l'électricité, de la radio et de la mécanique, 22,000; celui des mines, des moulins et des fonderies rassemblait 21,675 adhésions, sur un pied d'égalité avec les mineurs et les machinistes; les employés des établissements de salaison se groupaient au nombre de 17,000; enfin, les ouvriers du caoutchouc étaient organisés au nombre d'environ 10,000 .

Point n'est besoin de dire que, en ce qui concerne ces industries, le syndicalisme industriel a réalisé en dix ans une véritable révolution. Là où n'existait auparavant qu'un néant d'organisation sur le plan industriel, on retrouve aujourd'hui de puissants syndicats qui traitent d'égal à égal avec les entreprises. En plusieurs cas, des citadelles de l'anti-syndicalisme sont devenues des châteaux forts du mouvement ouvrier. Pour sa part, le Congrès des métiers et du travail a connu peu de succès dans ses efforts pour syndiquer les non organisés, surtout parce que le principe qui sert de base à son organisation ne correspondait pas à la tâche. Le Congrès canadien du travail, lui, a remporté victoire sur victoire, pour la bonne raison que le fondement de son travail d'organisation s'adaptait fort bien à la besogne à réaliser.

Autre différence notable entre les deux Congrès: le Congrès canadien du travail est autonome, tandis que le Congrès des métiers et du travail ne l'est pas. Les deux Congrès sont constitués pirncipalement de syndicats internationaux, dont le quartier général est installé aux Etats-Unis. De plus, ils sont tous deux affiliés aux deux principaux organismes ouvriers des Etats-Unis, la Fédération américaine du travail (A.F.L.) et le Comité d'organisation industrielle (C.I.O). Mais à cela se limite la ressemblance!

Le Congrès canadien du travail s'appuie sur une solide tradition de syndicalisme purement canadien. Son président, M. A.-R. Mosher, est le fondateur et le président de l'un des syndicats purement canadiens les plus anciens et les plus nombreux, la Fraternité canadienne des cheminots et d'autres employés du transport. Le Congrès canadien du travail est issu de la fusion du Congrès pancanadien du travail (All-Canadian Congress of Labour) et de succursales canadiennes de syndicats du C.I.O. Et le Congrès pancanadien du travail (All-Canadian Congress of Labour) n'a consenti à l'amalgation qu'à une condition: le nouveau Congrès, et de même les syndicats internationaux en faisant partie, devaient posséder leur autonomie complète.

Le Congrès ne doit ni sa formation, ni sa charte au C.I.O. Il ne se réclame d'aucune attache avec le C.I.O., ne lui paie aucune redevance et ne subit en aucune façon sa tutelle, qu'il s'agisse de questions d'ordre interne ou externe. Le Congrès peut s'affilier des syndicats qui ont par ailleurs des affinités avec des syndicats du C.I.O.: il l'a déjà fait. Il peut aussi refuser de reconnaître l'affiliation de syndicats du C.I.O., ce qu'il a d'ailleurs déjà fait. Les conflits de juridiction qui s'élèvent entre ses syndicats internationaux affiliés sont réglés, non pas par le C.I.O., mais bien par le Congrès canadien du travail! Sur un comité exécutif de neuf membres, trois - y compris le président appartiennent à des syndicats uniquement canadiens; deux - dont le secrétaire-trésorier - sont membres des United Mine Workers of America, (non affiliés aux Etats-Unis); quatre autres sont membres de syndicats qui, à cause de leurs syndiqués américains, sont affiliés au C.I.O. et lui paient leur cotisation, alors qu'en égard à leurs membres canadiens, ils sont affiliés au Congrès canadien du travail et lui versent leur quote-part.

La complète indépendance du Congrès canadien du travail vis-à-vis la tutelle du C.I.O. apparaît peut-être avec le plus d'évidence dans l'histoire de ses relations entre les United Mine Workers. Lors de la formation du Congrès canadien du travail, les U.M.W. faisaient partie du C.I.O. aux Etats-Unis : car son président international, M. Lewis, se trouvait en même temps président du C.I.O. En 1942, les U.M.W. se détachèrent du C.I.O., mais continuèrent leur adhésion au Congrès canadien du travail. En 1946, les U.M.W. s'affilièrent à la Fédération américaine du travail (A.F.L.), mais n'en demeurèrent pas moins avec le Congrès canadien du travail. En 1947 enfin, les U.M.W. abandonnaient la Fédération américaine du travail, mais demeuraient toujours au Congrès canadien du travail. Pendant six ans, les U.M.W. et les syndicats du C.I.O. aux Etats-Unis se sont livré une lutte sans merci; et pourtant, les succursales canadiennes des mêmes organismes ont continué leur pacifique coopération au Congrès canadien du travail. 
Le Congrès des métiers et du travail a, lui aussi, ses syndicats uniquement canadiens, de même qu'un puissant syndicat international, celui des machinistes, qui n'a pas d'affiliation aux EtatsUnis. Quelques années durant, son secrétaire-trésorier, M. Pat Sullivan, appartint à un syndicat purement canadien, celui des marins; et son président, M. Bengough, est membre du syndicat des machinistes. Fait digne de mention: il y a environ un an, quand la Fédération américaine du travail s'essaya à forcer la main au Congrès des métiers et du travail pour assurer l'expulsion des machinistes, la tentative s'avéra infructueuse.

D'autre part, le Congrès des métiers et du travail, de par sa constitution même, ne peut accepter l'affiliation de tout syndicat qui fait déjà le jeu avec la Fédération américaine du travail; et toute querelle de juridiction entre des syndicats de la Fédération américaine du travail affiliés au Congrès des métiers et du travail est réglée par la Fédération, et non par le Congrès. Le Congrès canadien du travail lui-même est un produit dérivé du manque d'autonomie du Congrès des métiers et du travail. Quand la Fédération américaine du travail expulsa les syndicats du C.I.O. aux Etats-Unis, le Congrès des métiers et du travail se montra fort peu disposé à imiter le geste, et en retarda l'exécution aussi longtemps qu'il le put. Mais devant l'insistance de la Fédération américaine, le Congrès dut céder. Autrement, c'était la rupture avec les syndicats de la Fédération américaine du travail. Plus récemment, dans le cas des machinistes, il ne fut pas question d'un organisme central rival ou du syndicalisme à double filiation; dans le cas du C.I.O., il en fut bien question!

Autre distinction très nette entre les deux Congrès: leur attitude devant l'action politique ouvrière. Le C.M.T. se refuse à adopter aucun parti politique. De son côté, le C.C.T. a endossé, en 1943, le programme du C.C.F. comme étant « l'arme politique du travail au Canada », avec recommandation à tous ses syndicats, affiliés ou à charte, d'emboîter le pas avec le C.C.F. A chacune de ses grandes réunions, depuis 1943, le C.C. T. a conservé et exprimé la même attitude; même qu'il a institué des comités d'action politique pour la concrétiser. Plusieurs chefs de syndicats du C.C.T. ont été candidats et même députés aux diverses Législatures provinciales. Encore, la Fédération ontarienne du travail (section ontarienne du C.C.T.) a joué un rôle très actif lors des récentes élections en Ontarì. tuée?

Mais pourquoi une différence aussi accen-

Il importe de souligner comme premier facteur l'influence des Etats-Unis sur les syndicats canadiens. Ce qui permet encore d'expliquer pourquoi seulement un nombre relativement restreint de syndicats du C.C.T. ont mis à profit la recommandation de s'affilier au parti cécéfiste. La politique de l'A.F.L. et du C.I.O., c'est la politique de Gompers : récompenser les amis du travail et punir les ennemis, sur le plan personnel.

Cette attitude se comprend assez bien aux Etats-Unis. Le président et son cabinet sont en dehors du Congrès américain, et n'ont aucun compte à lui rendre. Le président et les deux Chambres sont élus pour des mandats d'une durée fixe, et cette durée n'est pas la même pour les trois. Il ressort de ce fait que l'administration peut fort bien - comme c'est le cas présentement - appartenir à un parti politique différent de celui de la majorité des sénateurs ou des représentants. Mais pas plus le Sénat que la Chambre des représentants, même ligués dans leur tentative, ne peuvent se défaire du président avant l'expiration de son mandat, à moins d'une mise en accusation, cas qui se présente pour ainsi dire jamais.

Pour sa part, le président ne peut se débarrasser des sénateurs ou des représentants avant l'expiration de leur mandat. Et qui plus est, en vertu du mode particulier d'élection du président, il est parfaitement possible à un président amérisain leader de tel parti de faire face à un Sénat ou à une Chambre de représentants - ou même aux deux à la fois - à majorité politiquement hostile; et cela, durant tout le cours de son mandat. Les élections présidentielles se tiennent tous les quatre ans, alors que les élections à la Chambre se font tous les deux ans : ainsi, il arrive souvent que le président, appartenant à tel parti, ait à travailler avec une Chambre hostile durant la moitié de son mandat. Le Congrès peut bloquer le président; le président peut enrayer l'action du Congrès; de sorte que ni l'un ni l'autre ne peuvent réaliser efficacement leur politique. Le Congrès peut rejeter - il le fait souvent $l-$ les bills du président, et celui-ci doit se soumettre bon gré, mal gré. Et cette obstruction peut se produire même quand le propre parti du président a la majorité dans les deux Chambres! Son propre parti peut faire bloc contre lui et voter contre ses bills : et le président et le Congrès continuent de se maintenir comme si rien n'était arrivé ... 
Au Canada, le système est totalement différent. Le premier ministre et son cabinet font partie intégrante du Parlement et lui rendent leurs comptes. Ils ne sont pas élus en qualité de ministres, mais bien en qualité de députés, et aucun d'eux n'a un mandat fixe. Le Parlement et les Assemblées provinciales ne peuvent demeurer en place plus de cinq ans - quoique les Parlements provinciaux puissent prolonger leur mandat, et l'aient déjà fait -, mais elles peuvent aussi ne durer que quelques mois. Aucun gouvernement au Canada n'a à faire face à une Chambre hostile pendant bien des semaines, car la Chambre peut renverser le gouvernement; et puis, le gouvernement peut ordinairement se défaire de la Chambre. Si la Chambre pose un vote de non-confiance dans le gouvernement ou rejette un des bills importants, ou bien le gouvernement démissionne et fait place à un nouveau gouvernement qui gagne l'appui de la Chambre existante, ou bien c'est le gouvernement qui dissout le Parlement pour établir une nouvelle Chambre qui lui soit favorable.

Ce qui fait que la politique de Gompers est inconvenante et futile au Canada. Notre système élimine pour ainsi dire tous les membres indépendants et les «lâcheurs 》. Pour se servir des termes mêmes des as de l'opérette, Gilbert and Sullivan :

« When in that House M.P.'s divide.

«If they've a brain and cerebellum too, « They've got to leave that brain outside, « And vote just as their leaders tell'em to. 》

En d'autres mots, quand une discussion ou un vote s'amorcent en Chambre, les députés doivent oublier la présence et les exigences criantes de leur cerveau pour voter dans la ligne de leurs chefs. Car les députés qui feraient bloc contre leur chef risqueraient de placer au pouvoir le parti adverse et de perdre leur propre siègel

Aux Etats-Unis, les représentants et les sénateurs votent sur chaque bill à la lumière de leurs vues personnelles. Ainsi, tel député mérite l'appui des travailleurs parce que ses antécédents en regard des bills favorables aux travailleurs sont «bons », alors que tel autre mérite le désaveu des ouvriers parce que ses états de service sont «mauvais ». Au Canada, les députés ne votent pas sur chaque bill d'après leurs idées personnelles. Ils votent avec le parti. Notre système ne fonctionnera pas sur une autre base que celle-là. Jean-Baptiste Poulin, député, n'a pas d' « antécédents", d'\& états de service» personnels. C'est son parti qui a les 《 antécédents 》.
Au Canada, par conséquent, il ne sert de rien de vouloir mener une action efficace par le truchement des députés en particulier. Le travail doit agir par l'intermédiaire d'un parti Il lui faut balayer les partis hostiles à la classe travailleuse et mettre au pouvoir le parti qui lui est favorable. En théorie, il pourrait s'emparer de l'un des vieux partis. Mais dans la pratique, la formule s'avère irréalisable, parce que le seul parti canadien à structure démocratique et qui soit contrôlé par ses membres, c'est le C.C.F. Les vieux partis américains tiennent régulièrement des assemblées pour choisir leurs chefs et façonner leur plate-forme politique. Nos vieux partis n'agissent pas en ce sens. Le parti conservateur est né en 1854. Il n'eut sa première assemblée nationale qu'en 1927, et depuis, il n'en a connu que deux. Le parti libéral, lui, a été fondé en 1873. Il eut sa première assemblée nationale en 1893 - et même alors, on ne put y choisir un chef - et depuis, il n'en a tenu qu'une, en 1919. Et maintenant, le parti libéral est sur le point d'avoir sa $3 \mathrm{e}$ assemblée nationale. Dans l'ensemble, les vieux partis n'ont de congrès, nationaux ou provinciaux, qu'à la mort ou à la démission du grand chef. Aucun chef libéral ou progressiste-conservateur n'a jamais à descendre dans le « rang 》 de son parti à des intervalles réguliers pour rendre compte de son administration et se soumettre à une nouvelle élection. La politique du parti - sauf à des intervalles irréguliers et généralement longs - est déterminée par un petit groupe de chefs.

De son côté, le C.C.F. tient un congrès national tous les deux ans, et des congrès provinciaux chaque année. Ces congrès décident de la politique à suivre et élisent les chefs du parti. Les chefs sont responsables de leurs gestes politiques devant ces congrès. Chaque fois, ils doivent se faire réélire pendant ces congrès.

De plus - et c'est là un fait d'importance à souligner - le C.C.F. est le seul des grands partis canadiens à prévoir l'affiliation des syndicats com. me tels. Donc, au Canada, une action politique ouvrière qui soit efficace ne signifie pas seulement une action à travers un parti, mais pleinement une action à travers un nouveau parti.

Ce sont là les raisons pour lesquelles le Congrès canadien du travail - en dépit de la puissante influence de la tradition américaine - a préféré suivre la tradition des syndicats anglais et a endossé le C.C.F.. C'est aussi pourquoi plusieurs syndicats locaux des deux Congrès se sont affiliés au C.C.F. 
Un quatrième différence importante entre les deux Congrès, c'est leur attitude réciproque devant le communisme. Point n'est besoin de dire qu'aucun des deux Congrès n'est dominé par des communistes ou par des pro-communistes. D'autre part, il est indiscutablement vrai qu'aux deux Congrès se sont affiliés de puissants syndicats dominés par des communistes : ce fait se vérifie plus souvent dans le C.C.T. que dans le C.M.T. Chose assez paradoxale, cependant, le Congrès canadien du travail a adopté des positions beaucoup plus solides que le Congrès des métiers et du travail, en face du communisme. Les communistes se sont opposés avec acharnement à la décision d'endosser le C.C.F., et à diverses reprises ils ont tenté de la renverser; mais chaque fois ils ont été défaits à plate couture. Leurs candidats au comité exécutif ont presque chaque fois été battus par une marge énorme. Le dernier congrès national, tenu en octobre 1947, a adopté trois résolutions nettement anti-communistes. La première, portant sur la politique étrangère, fut emportée par 546 voix contre 165. La seconde, qui dénonçait le mouvement communiste comme tel, et la troisième, qui réaffirmait une autre fois l'adoption du parti cécéfiste, furent passées sans difficulté. Dans les dernières années, aucune résolution de ce genre n'a été adoptée lors des congrès nationaux du C.M.T.

Une raison peut expliquer ce dernier fait : le C.M.T., qui a pour principe de ne pas se compromettre dans la politique de parti, ne peut offrir une solution cohérente et positive en face du communisme. L'anticommunisme pur et simple est vain et stérile. Le Congrès canadien du travail l'a compris. Toujours il accompagne ses dénonciations du communisme et du programme communiste de suggestions positives qui peuvent contribuer à traiter les maux pour lesquels le communisme prétend fournir un remède. Toutes les résolutions anticommunistes du congrès national du C.C.T., en 1947 - et surtout celle qui endosse le C.C.F. - contiennent des propositions positives de cette nature. Au texte même de M. Després, les directeurs du Congrès « ne craignent pas d'affirmer leurs convictions socialistes et d'en propager le rayonnement dans les corps affiliés au Congrès. Recrutant ses effectifs dans les industries à production massive, le Congrès du Travail est conscient de la nécessité de maintenir l'embauchage intégral dans les industries de base. De tous les groupements ouvriers, c'est certes celui qui insiste le plus sur la nécessité d'une transformation radicále de notre régime économique et social.»

L'attitude très nette du C.C.T. en face du communisme s'explique encore par le caractère de son secrétaire-trésorier, M. Pat Conroy. M. Conroy, qui vient de recevoir de l'université St-FrançoisXavier le titre honorifique de Docteur en Droit, est un catholique convaincu, et un démocrate des pieds à la tête. Il est aussi un membre du C.C.F., convaincu qu'il est que ce parti offre le meilleur espoir de réaliser au Canada ce que l'université St-François-Xavier appelle «l'héritage divin des ouvriers et une conception plus chrétienne de la société économique. 》 En vertu de sa charge, mais plus encore par son intégrité, son courage, sa patience et sa tolérance, par sa compétence remarquable, M. Conroy exerce une influence décisive dans l'élaboration de la politique du C.C.T.

Une cinquième différence entre les deux Congrès réside dans leur relation avec la Fédération mondiale des unions ouvrières (World Federation of Trade Unions). Les deux Congrès ont participé à la conférence qui a décidé l'institution de la Fédération. M. Pat Sullivan, alors secrétaire-trésorier du C.M.T., fut membre du comité provisoire établi par la conférence. Mais le C.M.T. n'a jamais fait partie, par la suite, de la Fédération, et le C.C.T. est présentement le seul membre canadien de la Fédération. M. Conroy est membre du comité exécutif et du conseil de la Fédération. Il a joué un ròle de premier plan lors des récentes assemblées de la Fédération, tenues à Rome. En vérité, c'est grâce à lui si le Canada est représenté à l'exécutif : la constitution initiale de l'organisme mondial laissait le Canada sans représentation.

\section{Tarmes de callabaration patranale-amurière}

Le Rapport du troisième Congrès des relations industrielles de Laval (1948) contenant le texte des conférences et des délibérations est maintenant en vente au prix de $\$ 1.50$ (franco). Sujets traités: La convention collective ( $\mathrm{Me}$ Pau Lebel); La formation professionnelle (Gabriel Rousseau); Les comités d'entreprises (Raymond Gérin); La sécurité du travail, la médecine et l'hygiène industrielles (Dr Wilfrid Leblond); Théologie du travail (T.R.P. Georges-Henri Lévesque o.p.). 
Deux autres différences entre les Congrès, peut-être minimes présentement, mais qui peuvent s'accroître à la longue, ont rapport à la recherche et à l'éducation des travailleurs. Dans ces deux domaines, le C.C.T. s'est montré beaucoup plus actfi que le C.M.T.

Six syndicats du C.C.T. - les ouvriers de l'acier, les mineurs (district 26), les employés des établissements de salaison, les ouvriers de l'électricité, de la radio, et de la mécanique, les ouvriers du bois et les ouvriers du caoutchouc - possèdent des départements de recherches bien à eux. De même pour la Fédération ontarienne du travail. En outre, le Congrès a lui-même un département qui occupe continuellement une équipe de quatre personnes. On y imprime chaque mois un bulletin, on y fournit toutes les informations désirables et on y rédige des communiqués pour le Congrès, pour ses directeurs et pour les unions affiliées ou à charte. Le C.M.T. n'a rien de tel, et seulement un de ses syndicats possède un département de recherches, au Canada.

Le C.C.T. patronne aussi un comité d'éducation fort actif, qui déjà a organisé avec succès deux sessions d'études pour les travailleurs.

La première a consisté en un cours d'été de deux semaines au lac Couchiching, en Ontario, à la fin de juillet 1947. La seconde fut une semaine d'études à Ajax, encore en Ontario, en janvire 1948, avec la coopération de l'université de Toronto. On pense déjà à d'autres sessions de ce genre. Le C.M.T. n'a rien organisé de semblable. Mais il est encourageant de souligner que les deux Congrès, la Confédération des travailleurs catholiques du Canada et les Fraternités indépendantes de cheminots coopèrent avec l'université de Montréal dans l'établissement d'un institut du travail qui serait organisé dans la province de Québec.

Ces différences entre le Congrès des métiers et du travail et le Congrès canadien du travail sont importantes. Mais l'unité fondamentale des buts et des méthodes a aussi son importance. Les deux Congrès ont foi en la démocratie, et la réalisent dans la pratique. Ils veulent tous deux travailler à l'ombre de la loi. Tous deux, ils désirent une société stable, bien ordonnée et progressive, qui aille de l'avant grâce à des conventions collectives libres, à des élections libres, grâce à une méthode qui n'ait pas peur des essais, qui comprenne les erreurs et qui permette les compromis. Les deux Congrès rejettent le totalitarisme sous toutes ses formes. En dépit de leurs différences, ils ont tous deux trouvé moyen de coopérer sur plusieurs questions importantes de bien commun - témoin leur pétition conjointe émise récemment pour le rejet de la Loi des unions ouvrières (Trade Union Act) de l'Ile-du-Prince-Edouard. On peut espérer que de plus en plus ils travailleront ensemble, dans le sens où saint Paul l'entend, avec «diversité dans les dons, mais dans un même esprit. »

\section{LE DROIT DES TRAVAILLEURS DE PARTICIPER ACTIVEMENT A LA VIE DE L'ENTREPRISE 1}

L'auteur part du point de vue que le capital et le travail sont nécessaires à l'existence de l'entreprise. D'après le libéralisme et d'après le marxisme la propriété revient à un seul ou à l'Etat, mais le travail n'est qu'un simple instrument de production donc en rapport purement mécanique avec la vie de l'entreprise.

En ce qui concerne l'administration de l'entreprise, tout en sauvegardant l'unité indispensable dans la direction, il faut accorder aux travailleurs le droit de prendre une part active au destin de l'entreprise. De même les travailleurs doivent participer au profit.

(1) Sommaire de l'article intitulé: "The right of Labor to participate actively in the life of enterprise" qu'on trouvera en page 10 de la partie anglaise de ce Bulletin.
L'union organique du capital et du travail qui constitue l'être physique de l'entreprise doit se refléter dans les rapports de ses sujets et créer parmi eux une communauté vivante.

Puisque le capital est une chose morte tandis que le travail est l'immédiate expression de la personne humaine, ce n'est pas la propriété du capital qui justifie la liberté du travail. C'est plutôt la liberté immanente au travail qui justifie, entre certaines limites, la propriété personnelle du capital. De même l'autonomie de l'entreprise dans ses rapports avec l'Etat ne peut être valablement soutenue que si le travail, en s'affirmant comme sujet, la défend comme sienne. 